\title{
Propagation Analysis for Wireless Sensor Networks Applied to Viticulture
}

\author{
Felipe Pinheiro Correia, ${ }^{1}$ Marcelo Sampaio de Alencar, ${ }^{2}$ Waslon Terllizzie Araújo Lopes, ${ }^{2,3}$ \\ Mauro Soares de Assis, ${ }^{4}$ and Brauliro Gonçalves Leal ${ }^{5}$ \\ ${ }^{1}$ Federal Institute of Education Science and Technology of Pernambuco, Petrolina, PE, Brazil \\ ${ }^{2} P P g E E$, DEE, CEEI, Federal University of Campina Grande, Campina Grande, PB, Brazil \\ ${ }^{3}$ Federal University of Paraíba, João Pessoa, PB, Brazil \\ ${ }^{4}$ Brazilian Committee of URSI, Rio de Janeiro, RJ, Brazil \\ ${ }^{5}$ Federal University of São Francisco Valley, Juazeiro, BA, Brazil
}

Correspondence should be addressed to Waslon Terllizzie Araújo Lopes; waslon@ieee.org

Received 28 August 2016; Revised 30 November 2016; Accepted 18 December 2016; Published 30 January 2017

Academic Editor: Lorenzo Luini

Copyright (C) 2017 Felipe Pinheiro Correia et al. This is an open access article distributed under the Creative Commons Attribution License, which permits unrestricted use, distribution, and reproduction in any medium, provided the original work is properly cited.

\begin{abstract}
Wireless sensor networks have been proposed as a solution to obtain soil and environment information in large distributed areas. The main economic activity of the São Francisco Valley region in the Northeast of Brazil is the irrigated fruit production. The region is one of the major agricultural regions of the country. Grape plantations receive large investments and provide good financial return. However, the region still lacks electronic sensing systems to extract adequate information from plantations. Considering these facts, this paper presents a study of path loss in grape plantations for a $2.4 \mathrm{GHz}$ operating frequency. In order to determine the position of the sensor nodes, the research dealt with various environmental factors that influence the intensity of the received signal. It has been noticed that main plantation aisles favor the guided propagation, and the vegetation along the secondary plantation aisles compromises the propagation. Diffraction over the grape trees is the main propagation mechanism in the diagonal propagation path. Transmission carried out above the vineyard showed that reflection on the top of the trees is the main mechanism.
\end{abstract}

\section{Introduction}

The term Precision Agriculture (PA) refers to a set of technologies used to understand the changes that occur during the plantation cycles [1]. These tools should allow better monitoring of the stages of agricultural production. In this context, Wireless Sensors Networks (WSNs) have been proposed as a solution to perform this monitoring $[2,3]$. The emergence of protocols and low-cost devices enable the deployment of the technology in those environments, mainly by allowing the supervision of large areas.

This research has been developed in the São Francisco Valley region, Northeast of Brazil. The main economic activity is irrigated fruit growing for export, and the production of the region reaches the foreign market, especially Europe, where sanitary and quality standards are strict [4]. As mentioned, WSNs are candidates to improve the production process, as they provide new ways to manage the harvesting areas, along with the fact that they do not need transmission wires. Therefore, the cost of deployment of WSNs may be cheaper, and more adequate, than wired networks.

One of the possible applications of this research is to collect soil moisture data. With the humidity data it is possible to develop an automatic irrigation system taking into account areas of soil that are most in need of water, avoiding the uniform irrigation of the plantation. The proposed idea can be seen in Figure 1, which represents nodes arranged in a moisture map.

However, before the implementation of a communication system, it is important to know the path loss due to different antennas settings, related to the presence of obstacles, for example. Factors such as the transmission power, vegetation density, and the occurrence of line-of-sight also influence the project $[5,6]$. 


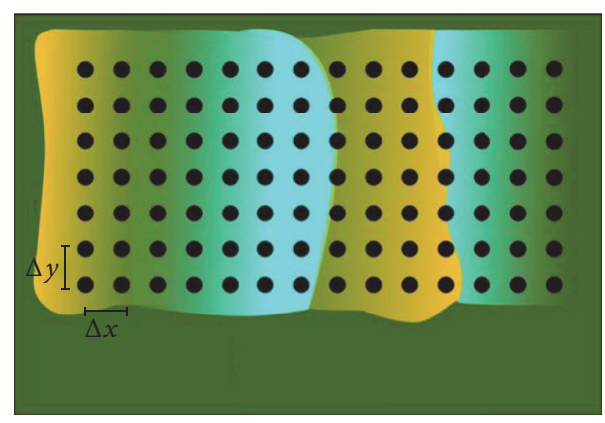

High humidity soil Low humidity soil

Ideal humidity soil Sensor node

FIGURE 1: Representation of sensors arranged in a humidity map that presents data by color.

Due to the lack of specific models, or parameters for the existing models in this type of application, this article presents an experimental study of the path loss found in grape plantations based on a in loco measurement campaign. Classical literature models were chosen and the parameters values were obtained empirically. From these values, discussions regarding the results have been performed. The objective of the research was to analyze the communication channel, predict the path loss, and evaluate the effects of the propagation phenomena on the signal intensity.

Based on the experiments performed in the same farm during several weeks, it has been noticed that the main plantation aisles favor the guided propagation. On the other hand, there was a considerable amount of vegetation along the secondary plantation aisles which compromised the propagation. Measurements taken following a diagonal path, in relation to the main aisle, have shown that diffraction by the grape trees is the main propagation mechanism. Transmission carried out above the vineyard, with both transceivers above it, has shown that reflection on the top of the trees is the main mechanism.

This paper is organized as follows. Section 2 presents related works in the area of WSNs in agriculture. Section 3 presents theoretical concepts regarding the propagation mechanisms. Section 4 shows the environment under study. The measurements are discussed in Section 5. Conclusions and future works are presented in Section 6.

\section{Wireless Sensor Networks and Agriculture}

The literature presents some works that deal with WSN applied to the agriculture, which motivated this research. The system proposed in [3] was developed for large-scale agricultural environments, using static sensor nodes with two goals: to collect information from newborn animals and to collect measurements of soil moisture. The nodes automatically read moisture data from the soil, typically in intervals of one minute, for each node. Then, the data is aggregated at the base to generate an updated profile for the entire pasture.
In [7], software and hardware for deployment of a WSN in Washington State have been developed. There are two networks, one to measure regional temperature and one to monitor plantation freezing. In the regional network, a master node is configured with multiple repeaters to build a backbone. The proposed network monitors the plantation and uses star topology. The biggest problems were encountered when the energy management and the switches were damaged during storms.

The Lofar Agro Project [8] has been developed to monitor microclimates in plantations. The purpose of monitoring is to combat the Phytophthora infestans bacteria that attack potato. The authors comment that humidity is an important factor in the development of diseases. A sensor network monitors, in addition to the humidity, the temperature and whether or not the leaves are wet, which are the main factors related to the Phytophthora infestation. The communications network has a weather station that measures light, air pressure, precipitation, wind direction, and strength.

Studies were performed in a cornfield [9] to evaluate the propagation characteristics with the IRIS Crossbow Inc. nodes. The influence of antenna height, the different directions within the planting area, the weather conditions (sunny day and rainy day), and transmitted power were considered. All factors were evaluated for the worst case: plantation with maximum height, maximum width of the stems, and long, thick leaves. It was possible to identify the conditions for increasing the coverage area by the sensor nodes.

A WSN was implemented to estimate the amount of water in the crop from the observed signal attenuation [10]. The nodes are deployed with the objective of collecting the signal. From the mean value of the power measured by the sensor nodes, the amount of water is estimated from the propagation model proposed by the authors. The advantage of this method is that the sensors are not required to obtain data.

Some studies using ZigBee are presented in [11-13]. In [11] an irrigation control system to optimize water usage is presented. In [12], a framework is proposed to monitor humidity, temperature, and lighting to control water consumption in agricultural areas. A WSN was developed in [13] using two modifications to the grid topology to improve network performance.

\section{Theoretical Background}

The WSNs suffer from various types of transmitted signal degradation. In wireless systems, the signal is attenuated in the path between the transmitter and receiver, according to the characteristics of the environment in which the electromagnetic wave propagates. These features include terrain deformations, obstacles between the antennas, and vegetation density. In addition, several propagation mechanisms influence the signal propagation, such as reflection, scattering, refraction, and diffraction [14].

3.1. Classical Propagation Models. The path loss is caused by the dissipation of power radiated by the transmitter, as well as by the effects of the propagation channel [15]. It can be defined 
as the power loss between the transmitter and receiver in a radio communication link.

There are two ways to model the path loss [16]: a priori modeling and measurement-based modeling. A priori models are appropriate for predicting path loss in places where it is difficult to perform measurements. The path loss is predicted using only a priori knowledge. The measurementbased models are related to methods to collect data and predict values of signal strength at locations where data cannot be collected.

3.1.1. Free Space Propagation. In free space, it is possible to devise a model with a flexible path loss exponent [16] for ideal isotropic antennas. The power (in $\mathrm{dBm}$ ) at the receptor is given by

$$
\begin{aligned}
P_{r}= & P_{t}+G_{t}+G_{r}+20 \log (\lambda)-10 \log \left(16 \pi^{2}\right) \\
& -10 \alpha \log (d)-10 \log (L),
\end{aligned}
$$

in which $P_{t}$ is the transmitted power, $G_{t}$ and $G_{r}$ are the transmitter and receiver gains, respectively, $\lambda$ is the wavelength of the transmitted signal, $d$ is the distance between transmitter and receiver, and $L$ is the system loss. The parameter $\alpha$ is adjusted using the least squares or least absolute residual regression models, for example, [17].

3.1.2. Ground Reflection Model. The ground reflection model, or the plane earth model $[18,19]$, is an a priori model. It considers a direct, and a reflected, ray with no obstacles between the transmitter and the receiver. The path loss is given by [19]

$$
L=-10 \log \left(G_{t} G_{r} \frac{h_{t}^{2} h_{r}^{2}}{d^{4}}\right),
$$

in which $G_{t}$ and $G_{r}$ are the transmitter and receiver gains, respectively, $h_{t}$ and $h_{r}$ are the heights of the antennas of the transmitter and the receiver, respectively, and $d$ is the distance between transmitter and receiver.

3.1.3. Log-Distance Model. The log-distance model attenuation parameter $n$ is obtained empirically. It is used for environments with shadowing, and the received power is given by [20]

$$
P_{r}=P\left(d_{0}\right)-10 n \log \left(\frac{d}{d_{0}}\right)+X,
$$

in which $P\left(d_{0}\right)$ is the average power measured at a reference distance, usually taken at 1 meter, $n$ is the distance loss exponent, and $X$ is a Gaussian random variable with mean $\mu$ and variance $\sigma^{2}$.

3.1.4. Propagation between Two Parallel Planes. The parallel plane guide is formed by two metal planes. There is a dielectric material in the middle with permittivity $\epsilon$. The electromagnetic wave propagates inside the structure in the $x$ direction (see Figure 2).

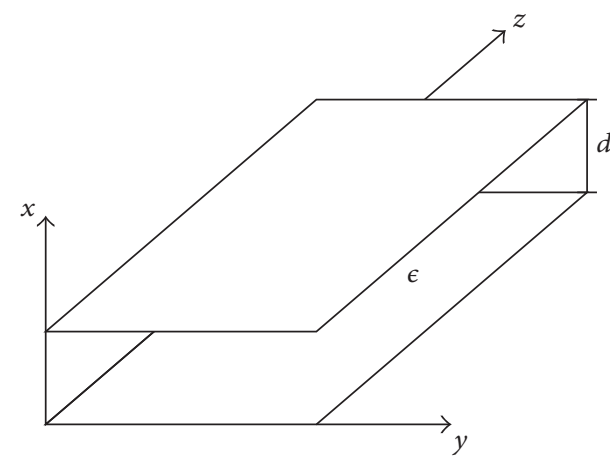

FIGURE 2: Waveguide diagram composed of two parallel planes.

Depending on the permeability and permittivity it is possible to have the value of the received power, inside parallel planes, very close to the received power in free space for the same distance; that is, the power along the parallel planes is a function of $d^{2}$.

3.1.5. Empirical Foliage Models. There are some models based on measurements to predict losses in different types of vegetation and at different frequencies. The exponential decay model proposed by Weissberger [21] is used when the path between the transmitter and the receiver is full of dense vegetation, composed of trees with low humidity. The loss due to vegetation is given by [21]

$$
L(\mathrm{~dB})= \begin{cases}1.33 f^{0.284} d^{0.588}, & 14 \mathrm{~m}<d \leq 400 \mathrm{~m} \\ 0.45 f^{0.284} d, & 0 \leq d<14 \mathrm{~m},\end{cases}
$$

in which $f$ is the frequency, in $\mathrm{GHz}$, and $d$ is the height of the trees, in meters.

An ITU-R recommendation was developed from measurements performed primarily in the UHF band. The distance between the transmitter and the receiver must be shorter than $400 \mathrm{~m}$, to allow the signal to propagate without the formation of a lateral wave. The excess attenuation is given by

$$
L(\mathrm{~dB})=0.2 f^{0.3} d^{0.6},
$$

in which $f$ is the frequency and $d$ is the distance.

According to these models, it is possible to verify that the excess loss due to vegetation can be represented by the expression [22]

$$
L(\mathrm{~dB})=A f^{B} d^{C} .
$$

From measurements taken in the environment, the parameters $A, B$, and $C$ can be obtained using optimization techniques to reduce the least square error.

3.2. Link Budget. The link budget [14] is used to obtain the signal strength at the receiver, considering all path losses between the transmitter and receiver. The received power, accounting for all gains and losses, is given by

$$
P_{r}=P_{t}+G_{t}-L_{\text {path }}+G_{r}
$$


in which $P_{r}$ is the received power in $\mathrm{dBm}, P_{t}$ is the power of the transmitter, $G_{t}$ is the transmitter gain, $L_{\text {path }}$ is the total path loss, and $G_{r}$ is the gain of the receptor.

\section{Environments}

The plantation studied covers an area of approximately 10 hectares and its average height is 2 meters. For this structure, tree trunks and wires are used to support the leaves and grapes in order to uniformize the height of the crop. This structure is called a vineyard, as can be seen in Figure 3.

Four height and position terminal settings have been identified within the plantation, which result in different dominant propagation mechanisms. The first case is when the terminals are below the vegetation top and positioned in the type A aisle. The second case occurs when the terminals are below the vegetation top, positioned in the type B aisle. When the terminals are below the top, and there are obstacles between the transmitter and the receiver, the third case is characterized. Finally, the fourth case occurs when both terminals are above the crop. Figure 6 presents the superior view in which type A aisle is also used as a reference axis. features:

The type A aisle is characterized, mainly, by the following

(i) The aisle is formed by the vines and stems which are evenly spaced on both sides.

(ii) The aisle is almost entirely covered by the leaves of the vines.

(iii) Vegetation along the aisle has a maximum height of $40 \mathrm{~cm}$.

(iv) There are areas where the ground vegetation has $5 \mathrm{~cm}$ height.

Type A aisle is presented in Figure 3. The distance between the vines is around $3 \mathrm{~m}$, and there is a larger amount of vegetation, since the vegetation along type $B$ aisle grows to a height of $90 \mathrm{~cm}$, approximately (Figure 4). There are also ground elevations along the rows. Furthermore, there is a large quantity of grapes throughout the path, as shown in Figure 5 . There are areas where the vegetation is about $5 \mathrm{~cm}$ height.

The diagonal environment is similar to the environment described for the type B aisle, except for the tree trunks positioned between the two devices. There is a lot of vegetation and there are elevations along the rows. There are grapes between nodes and there exist areas, where the vegetation is about $5 \mathrm{~cm}$ high. The vegetation that covers most of the area is supported by wire, by trunks of vines and wooden stakes.

4.1. Methodology. The experiments described in this section were performed in the Minuano farm, located $70 \mathrm{~km}$ from Petrolina city, in the state of Pernambuco, Brazil. The climate is classified as tropical semiarid, with low rainfall rates throughout the year, favoring the production of grapes. Besides the scarcity and irregularity of rainfall, the rains occur mainly in the summer, and there is strong evaporation, as a result of the high temperatures. The average temperature during the experiments was $36^{\circ} \mathrm{C}$.

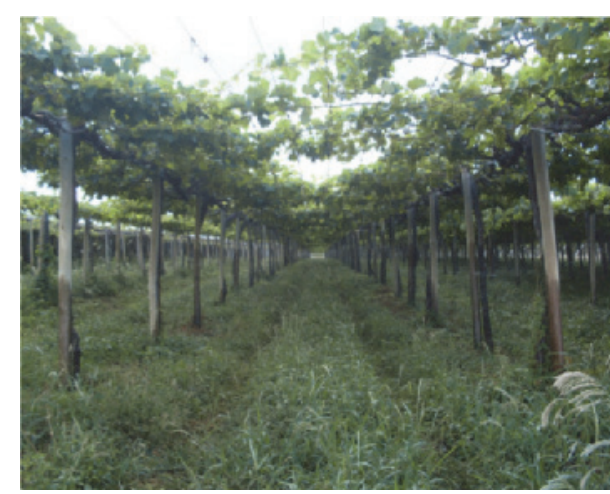

Figure 3: Photo of the vineyard used for the experiments.

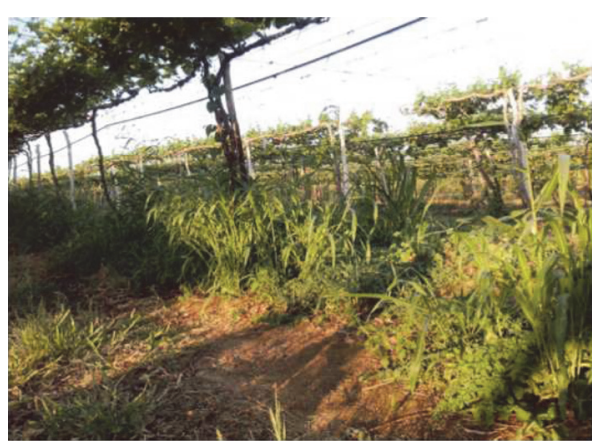

FIGURE 4: Photos of type B aisle vegetation, in the Vineyard used for the tests.

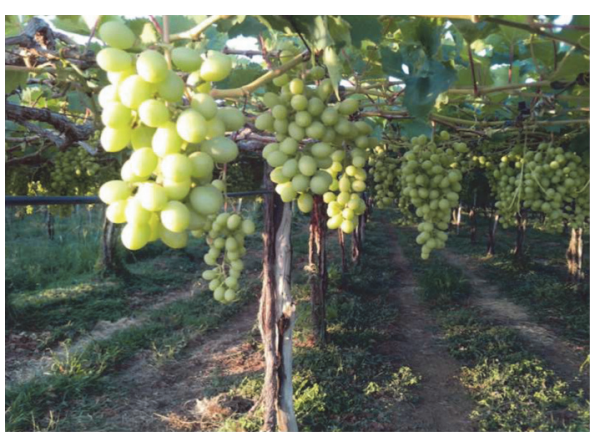

Figure 5: Photo of grapes along type B aisle.

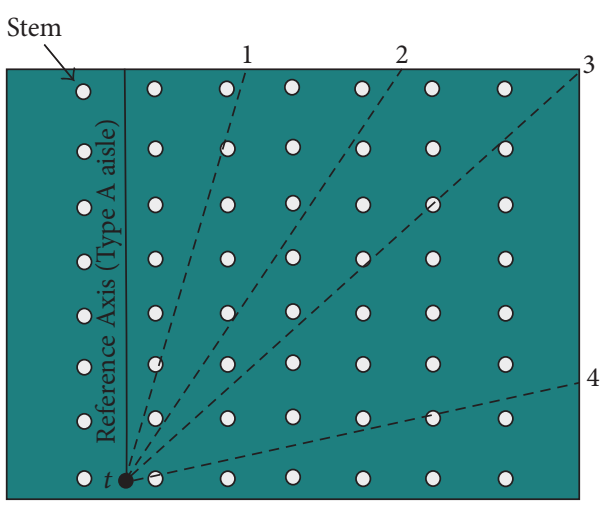

FIGURE 6: Superior view of the plantation, with the lines used to guide the measurements following the diagonals. 
A pair of XBee ZB Pro S2B nodes was used. The devices have $+18 \mathrm{dBm}$ of output power, $-102 \mathrm{dBm}$ sensitivity specified by the manufacturer, and the operating frequency is $2.4 \mathrm{GHz}$. The antenna is one dipole type. Both devices presented the same specifications, except that one was connected to a notebook, so that the data could be stored in a database. This terminal was configured as a coordinator.

One of the terminals (endpoint) was kept at the same position, and the other one (coordinator node) was strategically positioned along the plantation. At each point, the coordinator node requires one Received Signal Strength Indicator (RSSI) package of the endpoint. The measurement procedure was as follows:

(1) The coordinator (transmitter) is connected to a notebook and positioned at a distance of 1 meter from endpoint.

(2) The coordinator sends one package to the endpoint, requiring it to calculate the RSSI.

(3) The endpoint transmits the package with the computed value.

(4) Repeat steps (3) and (4) one hundred times.

(5) This procedure is repeated at different distances, until the power is low enough, or the plantation fence is reached.

(6) Data are analyzed using Gnuplot and MATLAB.

(7) One propagation model is chosen and adjusted for each case.

4.1.1. Measurements. The measurements taken along type A aisle were performed with the nodes positioned in the middle of the aisle. The receiver was installed at a height of $1 \mathrm{~m}$. First, the transmitter was installed at a height of $20 \mathrm{~cm}$ above the ground. One hundred signal intensity measurements were made at intervals of 50 meters. The experiment was repeated with the transmitter positioned at heights of $40 \mathrm{~cm}, 60 \mathrm{~cm}$, and $1 \mathrm{~m}$. These measurements were taken during the "in leave" plantation stage.

Other measurements were performed for different stages of the plantation, at a height of $1 \mathrm{~m}$. The transmitter was placed along type A aisle when the plantation did not have any leaves, only the wires that supported them covered the top of the plantation. In a second round of measurements, data were collected during the "in fruit" plantation stage.

At a distance of $400 \mathrm{~m}$, the end of the plantation was reached. However, comparing the power value at that distance with the sensitivity specified by the manufacturer, it is noticed that the range is larger than $400 \mathrm{~m}$. Any aisles long enough to perform the measurements beyond $400 \mathrm{~m}$ have not been found in this plantation.

Along type $\mathrm{B}$ aisle, measurements were performed with the nodes positioned in the middle of the aisle. The enddevice was kept at a fixed position at a height of $1 \mathrm{~m}$. The transmitter was installed at different heights, such as in type A aisle, $20 \mathrm{~cm}, 40 \mathrm{~cm}, 60 \mathrm{~cm}$, and $1 \mathrm{~m}$. It has been found in preliminary experiments that the range, in this case, is about $390 \mathrm{~m}$. One hundred signal strength measurements

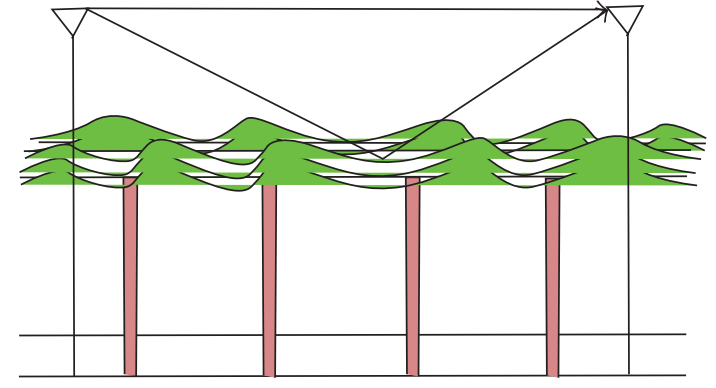

FIGURE 7: Diagram which represents the position of sensor nodes above the plantation.

were made at intervals of $25 \mathrm{~m}$ instead of $50 \mathrm{~m}$ to better observe the signal decay behavior.

As in type A aisle, measurements were limited, due to the size of the plantation, to a distance of $200 \mathrm{~m}$. Using a direct comparison between the power at the distance of $200 \mathrm{~m}$ and the device sensitivity $(-102 \mathrm{dBm})$, one realizes that the range goes beyond $200 \mathrm{~m}$. However, no type $B$ aisle long enough was found to perform measurements beyond $200 \mathrm{~m}$.

For the diagonal case, the measurements were performed following some angles, as shown in Figure 6. The enddevice was kept in a fixed position with a height of $1 \mathrm{~m}$. The transmitter is installed, first, at a height of $20 \mathrm{~cm}$ above the ground. One hundred signal strength measurements were made following the dashed lines forming 15, 30, 45, and 75 degrees (lines 1, 2, 3, and 4 of Figure 6, resp.) with type A aisle. The experiment was repeated with the transmitter at heights of $40 \mathrm{~cm}, 60 \mathrm{~cm}$, and $1 \mathrm{~m}$.

In some positions, when the signal strength was around $-97 \mathrm{dBm}$, the communication was not established. It was necessary to make several attempts until the data could be collected. Furthermore, in this case, the range is smaller than type A and type B aisles. This is an expected result because there are obstacles between the transmitter and the receiver.

Measurements performed with the coordinator above the plantation were performed, as illustrated in Figure 7. The end-device was installed at a height of $3 \mathrm{~m}$, positioned in the middle of the aisle. Then, the coordinator was moved along type $\mathrm{A}$ aisle at a height of $3 \mathrm{~m}$.

4.1.2. Least Absolute Residual Method. The least absolute residual (LAR) method was used to adjust the curves. It minimizes the sum of the deviation modules. The advantage of this method is that it is more robust against outliers [17].

Considering $y_{i}$ the power and $x_{i}$ the distance, for the unidimensional case, the method consists of determining $\beta_{0}$ parameter in

$$
y_{i}=\beta_{0}+\epsilon_{i}
$$

so that the absolute sum of the errors is minimized,

$$
\min _{\beta_{0}} \sum_{i=1}^{n}\left|\epsilon_{i}\right|=\min _{\beta_{0}} \sum_{i=1}^{n}\left|y_{i}-\beta_{0}\right| .
$$


The value of $\beta_{0}$ that minimizes the sum is the sample median, which explains the decrease of the outliers contribution. For linear regression,

$$
y_{i}=\beta_{0}+\beta_{1} x_{i}+\epsilon_{i}
$$

$\beta_{0}$ and $\beta_{1}$ parameters are calculated using linear programming to minimize [17]

$$
\min \sum_{i=1}^{n}\left|y_{i}-\beta_{0}-\beta_{1} x_{i}\right|
$$

\section{Interpretation of Measurements}

In order to measure the goodness of the model adjustments, the Root Mean Square Error (RMSE) has been used [17]:

$$
\mathrm{RMSE}=\sqrt{\frac{\left(\sum_{i=1}^{N}\left(x_{i}-\widehat{x}_{i}\right)^{2}\right)}{N}},
$$

in which $N$ is the number of points in the sample, and $x_{i}$ and $\widehat{x}_{i}$ are the measured and predicted values, respectively.

The determination coefficient $R^{2}$ is also used as a metric in the study of regression and is given by [23]

$$
R^{2}=\frac{\text { Explained Variation }}{\text { Total Variation }}=\frac{\sum_{i=1}^{N}\left(x_{i}-\bar{x}\right)^{2}}{\sum_{i=1}^{N}\left(\hat{x}_{i}-\bar{x}\right)^{2}}
$$

The total variance is the sum of the squares of the differences between the measured value and the average of the measured values. The explained variance is the sum of squares of the differences between each predicted value and the average of the measured values. This measure indicates how good is the regression, that is, how much the regression line approaches the collected data. The coefficient $R^{2}$ is in the range $[0,1]$. The closer to one, the better the regression line describes the behavior of the data.

5.1. Type A Aisle. Figure 8 shows the measurements of type A aisle, for Friis free space and plane earth models without correction factors. The vertical axis represents the average received power in $\mathrm{dBm}$ and the horizontal axis represents the distance between antennas.

The models were chosen for the first analysis because there are no obstacles between the terminals. It is observed, however, that the signal strength decay does not follow the plane earth model. One can verify this statement by averaging the differences between the value predicted by the plane earth model and the measured value. Thus, the average gain is equal to $7.87 \mathrm{~dB}$. Table 1 presents the RMSE values.

From the curves and RMSE values, it seems that the geometry of the plantation favors the guided propagation of the wave. This fact is evidenced mainly by the RMSE value for free space that is lower than the plane earth model. Graphically, it is also possible to notice this behavior. However, a dependency on the height is identified, caused by the reflected ray at $d=100 \mathrm{~m}$ (limit of the first Fresnel zone). In addition, two adjustments of the theoretical models were

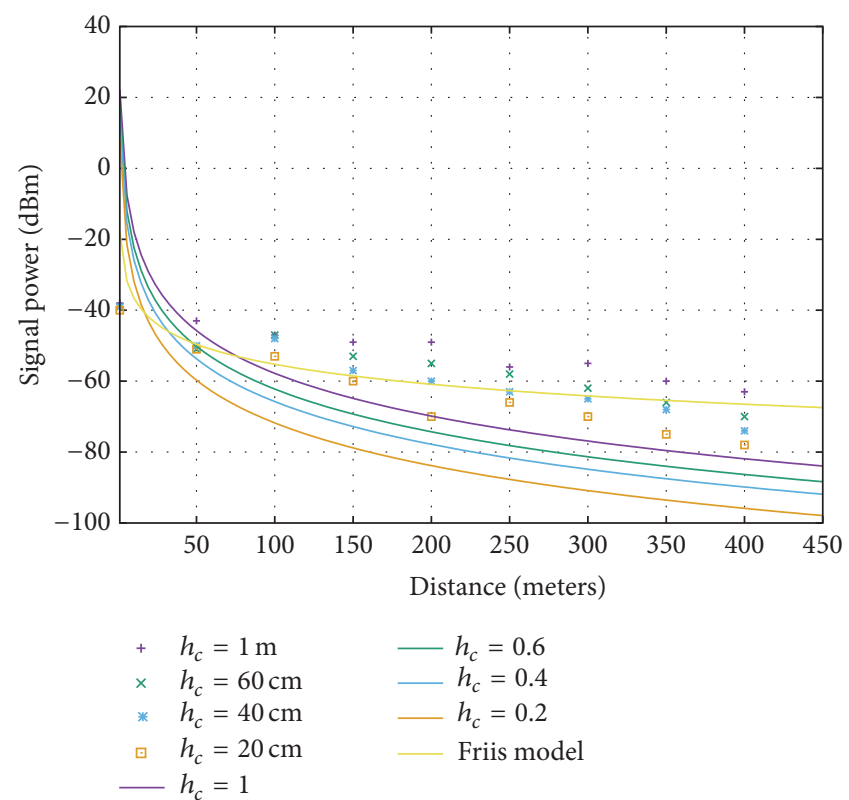

FIGURE 8: Received power $(\mathrm{dBm})$ as a function of distance, and Friis free space and plane earth models for type A aisle (linear scale for distance).

TABLE 1: RMSE for Friis free space and plane earth models for type A aisle.

\begin{tabular}{lc}
\hline Model, height & RMSE $(\mathrm{dBm})$ \\
\hline Friis & 9.7914 \\
Plane earth, 1 meter & 25.6181 \\
Plane earth, $60 \mathrm{~cm}$ & 21.1751 \\
Plane earth, $40 \mathrm{~cm}$ & 23.6352 \\
Plane earth, $20 \mathrm{~cm}$ & 28.8378 \\
\hline
\end{tabular}

made using the LAR method in MATLAB. The exponents of distance for both free space and the plane earth model were adjusted to minimize the RMSE. Changing the plane earth model, since the decay is not proportional to $d^{-4}$,

$$
\begin{aligned}
P_{r}= & P_{t}+G_{t}+G_{r}+20 \log \left(h_{t}\right)+20 \log \left(h_{r}\right) \\
& -10 n \log (d),
\end{aligned}
$$

in which $n$ is the adjusted distance exponent. The values obtained for the exponents are presented in Table 2, with the RMSE values for each case. The values of $P_{t}, G_{t}$, and $G_{r}$ are provided by the XBee manufacturer and are equal to $18 \mathrm{dBm}$, $2.1 \mathrm{~dB}$, and $2.1 \mathrm{~dB}$, respectively.

It can be seen from Table 2 that the Friis model, with the adjusted exponent, has an exponent close of two, with little improvement of the RMSE. Regarding the plane earth model, the RMSE decreases, indicating a better fit, but the decay is not proportional to the fourth power of the distance. Figure 9 presents the curves with the adjusted exponents.

Figure 2 shows the results for measurements done for different stages of the plantation at the height of $1 \mathrm{~m}$. Both "in fruit" and "in leave" cases are characterized by the waveguide effect, although, mainly because of spreading caused by the 
TABLE 2: Friis free space and plane earth models RMSE with adjusted exponents for type A aisle.

\begin{tabular}{lccc}
\hline Model, height & Exponent $(n)$ & RMSE $(\mathrm{dBm})$ & $R^{2}$ \\
\hline Friis & 1.87 & 9.35 & 0.95 \\
Plane earth, 1 meter & 3.29 & 6.96 & 0.98 \\
Plane earth, $60 \mathrm{~cm}$ & 3.25 & 6.95 & 0.99 \\
Plane earth, $40 \mathrm{~cm}$ & 3.25 & 6.28 & 0.96 \\
Plane earth, $20 \mathrm{~cm}$ & 3.25 & 6.68 & 0.94 \\
\hline
\end{tabular}

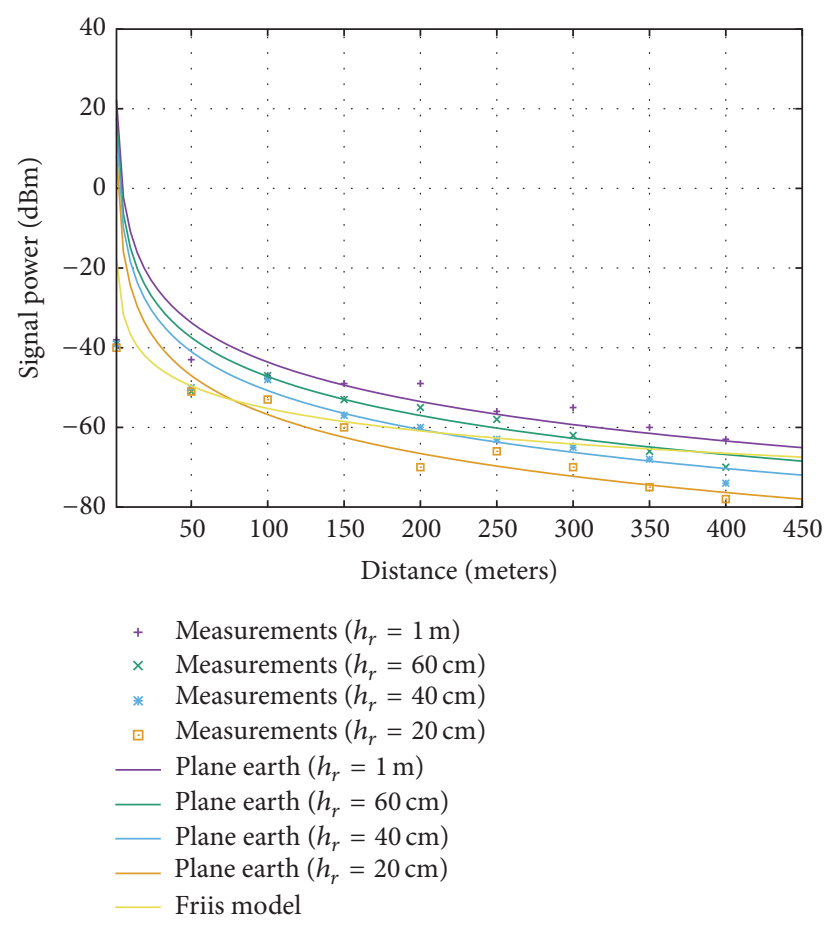

FIgURE 9: Received power $(\mathrm{dBm})$ as a function of distance for Friis free space and plane earth models with adjusted exponents for type A aisle (linear scale for distance).

water in the fruits, the attenuation is greater for the "in fruit" case. When the wave guide is unset, that is, when there are no leaves on top of the plantation, the signal power decays much faster and the plane earth model does not fit well. As the measurements do not have a logarithmic behavior, the linear regression was used instead; the following expression has been obtained:

$$
P_{r}=-39.82-0.1978 d .
$$

This result can be explained by spreading and absorption of the electromagnetic field caused by the wires. Table 3 presents the exponents of the models. It is important to highlight the difference of 0.36 between "in fruit" and "in leave" adjustments. The corresponding plot of the received power $(\mathrm{dBm})$, as a function of the distance, is presented in Figure 10.

5.2. Type B Aisle. As type B aisle is characterized by a higher density of vegetation, scattering and absorption cause losses that cancel the canalization effect. In Figure 11, it can be
TABLE 3: RMSE of the models used for different plantation states along type A aisle.

\begin{tabular}{lccc}
\hline Model & Exponent $(n)$ & RMSE $(\mathrm{dBm})$ & $R^{2}$ \\
\hline Plane earth, in fruit & 3.65 & 6.92 & 0.95 \\
Plane earth, in leave & 3.29 & 6.96 & 0.98 \\
Linear, no leaves & 0.1978 & 1.77 & 0.99 \\
\hline
\end{tabular}

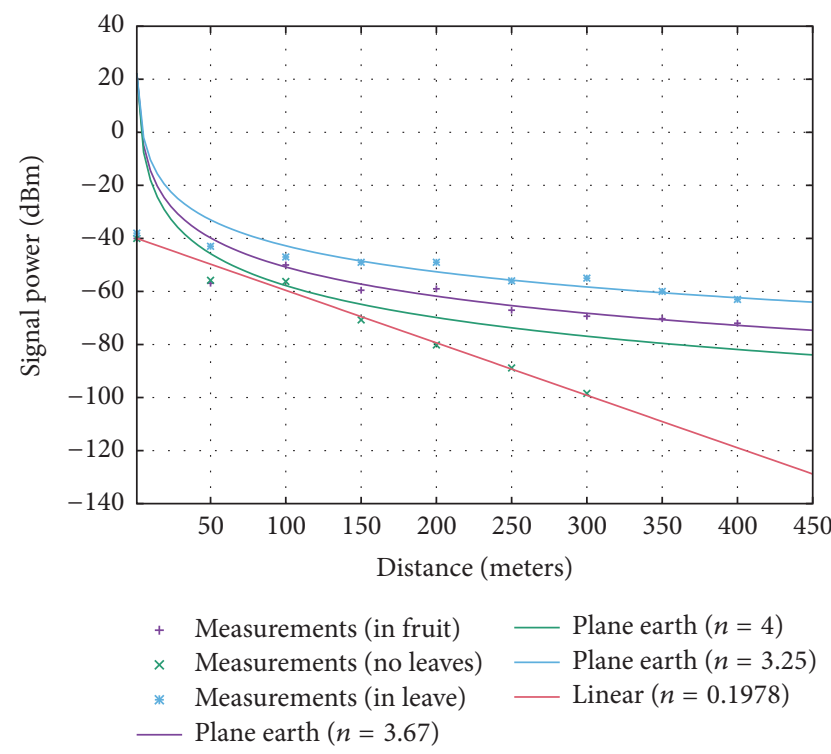

FIGURE 10: Received power $(\mathrm{dBm})$ as a function of distance for different stages (in leave, in fruit, and no fruit) of the plantation (linear scale for distance).

seen that the decay is faster for short distances and, as the transmitter moves away from the receiver, the power decays slowly, indicating a logarithmic behavior. Therefore, the log-distance model was used with some modifications. The attenuation is given by

$$
A=10 n \log \left(\frac{d}{d_{0}}\right)+X,
$$

in which $d$ is the distance, $d_{0}$ is the reference distance taken at 1 meter, and $n$ is obtained from the LAR method; $X$ is a Gaussian random variable with zero mean. $G_{r}$,

One modification is the substitution of $P\left(d_{0}\right)$ by $P_{t}+G_{t}+$

$$
A=P_{t}+G_{t}+G_{r}-P_{r}=C-P_{r}
$$

in which $C$ is given by

$$
C=P_{t}+G_{t}+G_{r}=22.2 \mathrm{dBm} .
$$

As this work does not address the small-scale fading, the $X$ term does not appear in the adjusted equations. Therefore, the received power is given by

$$
P_{r}=P_{t}+G_{t}+G_{r}-10 n \log (d) \mathrm{dBm} .
$$

The value of $n$ is obtained from the LAR method implementation provided by MATLAB and is equal to 4.537. The 


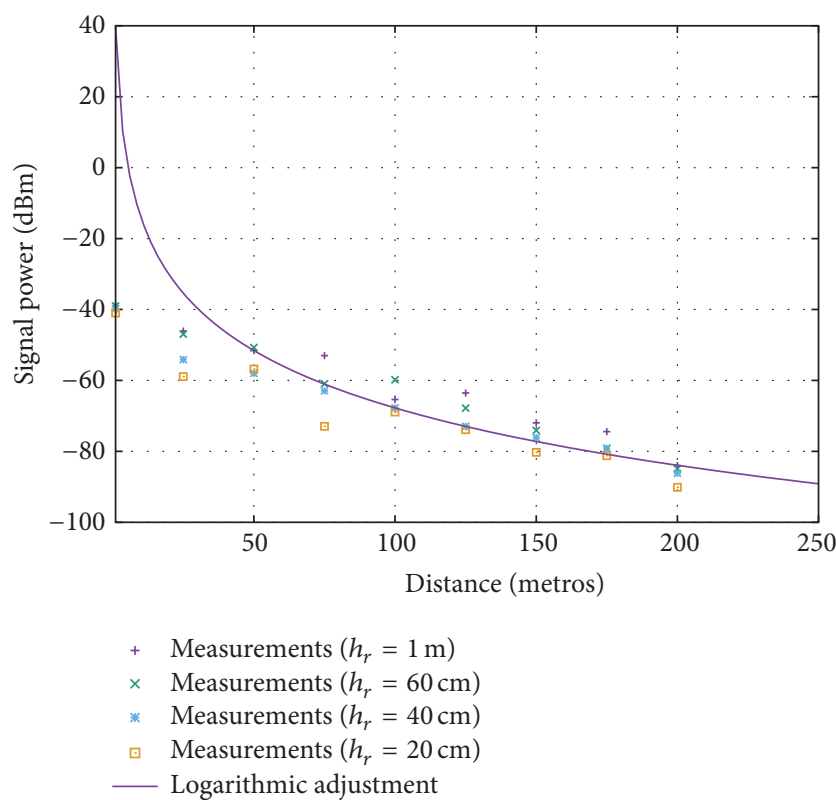

FIGURE 11: Received power $(\mathrm{dBm})$, as a function of distance and adjusted logarithmic equation (linear scale for distance).

resulting RMSE is equal to 3.567 , and $R^{2}$ equals 0.9399 . The curve obtained is shown in Figure 11, with the distance in a linear scale.

Another option to adjust the curves is to use an equation that includes the height, so that the model provides more parameters and lower RMSE. Start from the following expression:

$$
P_{r}=\left(P\left(d_{0}\right)+f\left(h_{r}\right)\right)-n d,
$$

in which $P\left(d_{0}\right)$ is the power at the reference distance (1 meter), $f\left(h_{r}\right)$ is expressed as a function of the receiver distance, $n$ is a constant, and $d$ is the distance. The values of $P\left(d_{0}\right)+f\left(h_{r}\right)$ and $n$ are obtained using the LAR method. Considering $h_{r}=1 \mathrm{~m}$ the reference height, it is possible to obtain the following formula, for $h_{r}=1$ :

$$
P_{r}=-39.82-0.2115 d \text {. }
$$

Therefore, $P\left(d_{0}\right)+f\left(h_{r}\right)=-39.82$. Following the same steps for other heights,

$$
\begin{array}{ll}
P\left(d_{0}\right)+f\left(h_{r}\right)=-41.43, & \text { para } h_{r}=0.60 \\
P\left(d_{0}\right)+f\left(h_{r}\right)=-45.16, & \text { para } h_{r}=0.40 \\
P\left(d_{0}\right)+f\left(h_{r}\right)=-48.17, & \text { para } h_{r}=0.20 .
\end{array}
$$

The adjusting equation chosen for $f\left(h_{r}\right)$ was

$$
f\left(h_{r}\right)=a e^{b h_{r}},
$$

in which $a$ and $b$ are obtained using the LAR method and are equal to -12.07 and -2.41 , respectively. It was possible to find a complete equation, given by

$$
\begin{aligned}
& P_{r}=\overline{P\left(d_{0}\right)}-n d+f\left(h_{r}\right) \\
& P_{r}=-39,69-0,2115 d-12,07 e^{-2,41 h_{r}},
\end{aligned}
$$

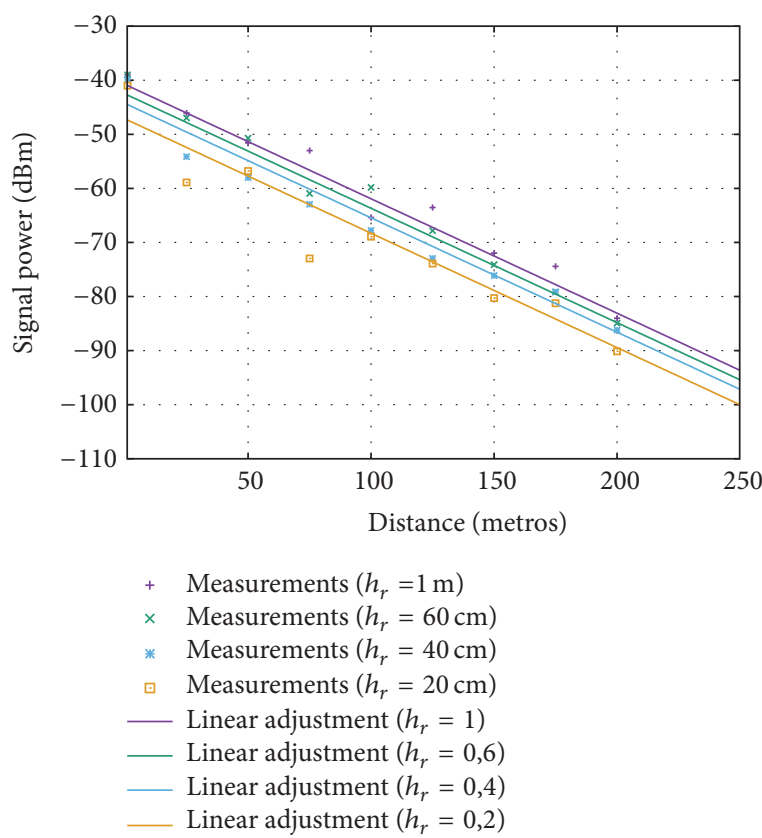

FIGURE 12: Received power ( $\mathrm{dBm})$, as a function of distance and the adjusted linear equation (linear scale for distance).

TABLE 4: RMSE and $R^{2}$ of the adjusted linear equation.

\begin{tabular}{lcc}
\hline Receptor height & RMSE $(\mathrm{dBm})$ & $R^{2}$ \\
\hline$h_{r}=1 \mathrm{~m}$ & 2.43 & 0.97 \\
$h_{r}=0.60 \mathrm{~cm}$ & 2.20 & 0.98 \\
$h_{r}=0.40 \mathrm{~cm}$ & 2.90 & 0.96 \\
$h_{r}=0.20 \mathrm{~cm}$ & 4.64 & 0.90 \\
\hline
\end{tabular}

in which $P\left(d_{0}\right)$ is the average received power at the reference distance. The graph in linear scale for the distance is shown in Figure 12. Table 4 presents the RMSE and $R^{2}$ values.

5.3. Diagonal. First, a comparison between the Weissberger model and the recommendation of the ITU-R for the UHF band regarding all performed measurements along the diagonal has been performed. The equations for this case, including the excess loss by vegetation, are

$$
\begin{aligned}
& P_{r}=A_{e l}-1,33 f^{0,284} d^{0,588} \\
& P_{r}=A_{e l}-0,2 f^{0,3} d^{0,6},
\end{aligned}
$$

in which $A_{e l}$ is the attenuation in free space, $f$ is the frequency, and $d$ is the distance. The plot of the measurements and the model are presented in Figure 13.

Although the measurements made by Weissberger have been taken in different environments, the proposed equation presents a reasonable fit in this case. However, in order to reduce the RMSE value, the parameters $A, B$, and $C$ of the excess attenuation were adjusted. In addition, the exponent of log-distance model and a linear fit have been determined. The curves for the adjusted models are presented in Figure 14. 


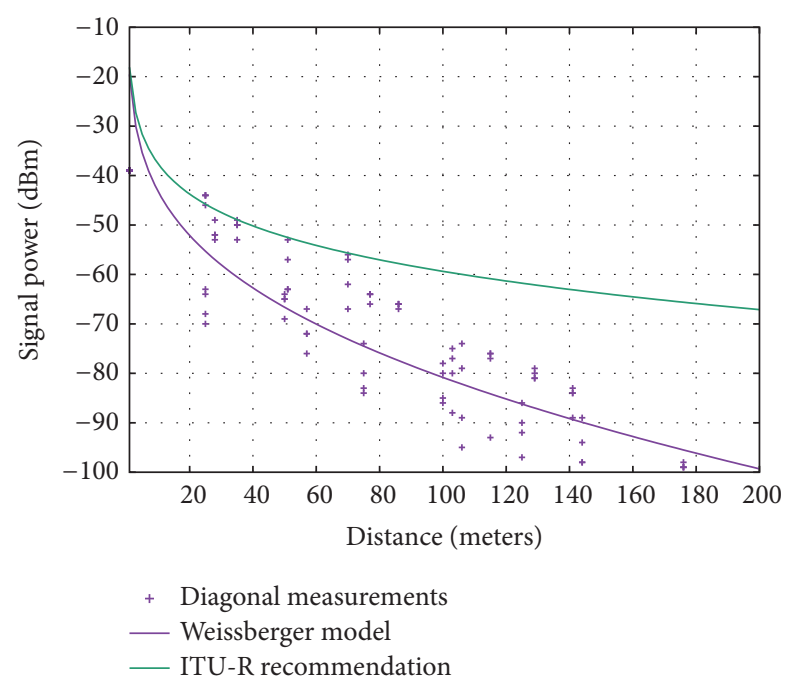

FIGURE 13: Received power ( $\mathrm{dBm})$, as a function of distance, Weissberger and ITU-R equations (linear scale for distance).

TABle 5: Parameters, RMSE values and $R^{2}$ for Weissberger, and logarithmic and linear equations.

\begin{tabular}{lccc}
\hline Model & Parameters & RMSE $(\mathrm{dBm})$ & $R^{2}$ \\
\hline \multirow{3}{*}{ Modified Weissberger } & $A=0.08$ & & \\
& $B=1.96$ & 10.98 & 0.67 \\
& $C=0.83$ & & \\
\hline Logarithmic & $n=5.06$ & 2.69 & 0.98 \\
\hline Linear & $n=0.36$ & 7.64 & 0.83 \\
\hline
\end{tabular}

Table 5 presents the exponents, the RMSE, and $R^{2}$ values obtained.

5.4. Terminals above the Vineyard. When the two terminals are above the plantation, the electromagnetic field is reflected on the top of the plantation structure formed by the metallic wires and leaves. Thus, the plane earth model is used, considering the heights of the nodes equal to $1 \mathrm{~m}$, instead

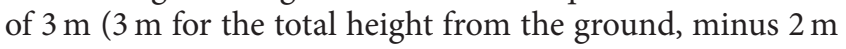
height of the piles). In this situation, until a distance of 100 meters is reached, the propagation occurs in free space, since the clearance of the route is larger than $0.6 R$, in which $R$ is the radius of the first Fresnel zone.

For measurements taken up to a 100 meters, the gap relative to the top structure is approximately $0.6 R$; that is, the plane earth and free space model leads to the same attenuation value. For this distance, the main propagation mechanism is the plane earth with the reflected ray on the top of the plantation. The RMSE for the plane earth model, with the adjusted exponent, equals 5.64. The value of the exponent is equal to 4.017. The graph of Figure 15 shows the measurements collected with the terminals above the plantation following type A aisle and Friis and plane earth models.

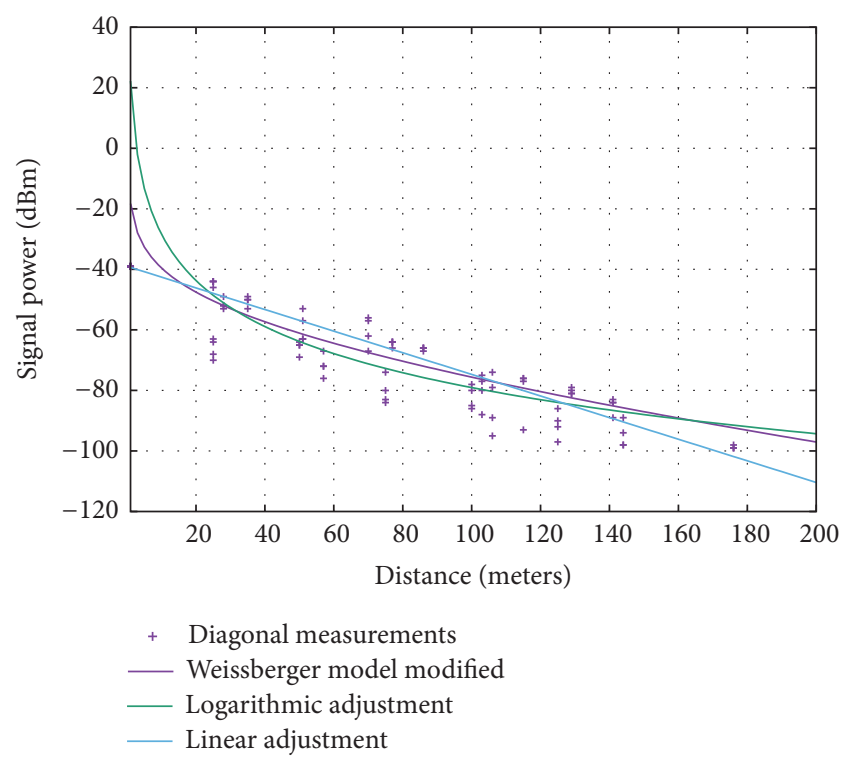

FIGURE 14: Received power (dBm), as a function of distance, modified Weissberger model, and linear and log-distance adjustments (linear scale for distance).

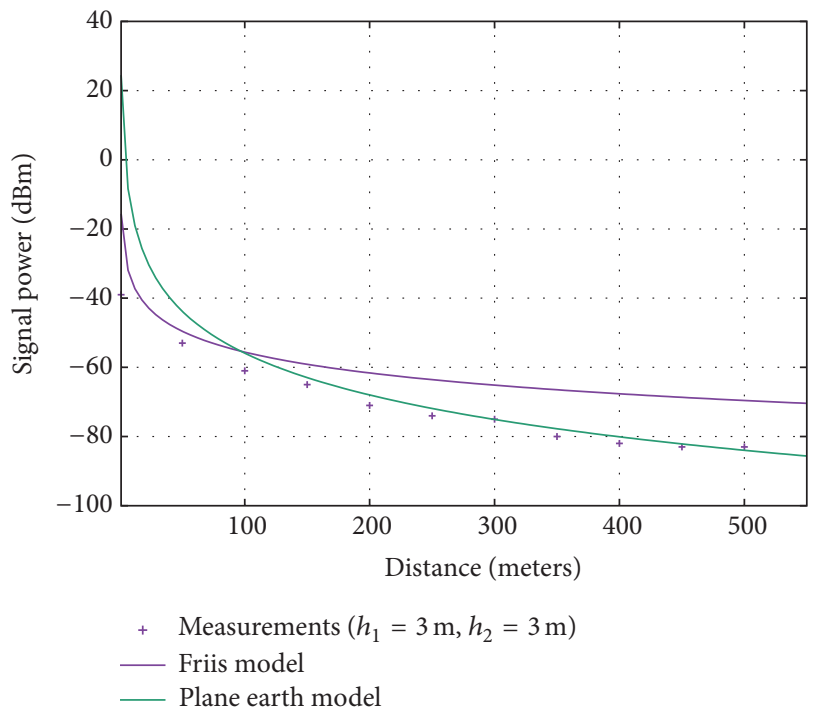

FIGURE 15: Received power $(\mathrm{dBm})$ as a function of distance, Friis free space, and plane earth models for the case in which the terminals are above the plantation (linear scale for distance).

\section{Conclusions and Future Works}

This paper presents a study of path loss in grape plantations for a $2.4 \mathrm{GHz}$ operating frequency. The main contributions of this research are the identification and interpretation of the propagation phenomena in the studied environment, as well as the methodology adopted to obtain the data parameters. The researched environment has characteristics that favor certain propagation mechanisms depending on the situation.

The region was divided into four areas characterized by different dominant propagation mechanisms. Type A aisle 
is free of obstacles and forms a structure that favors the propagation as a parallel plate waveguide, although there is a dependence of height. In type B aisle, scattering and absorption caused by vegetation which is denser increase the signal attenuation compared with the propagation along type A aisle.

The diagonal propagation is affected by multiple diffraction around the trunks of vines, which raises the distance exponent. When the two transmitters are above the plantation, the top structure reflects the signal.

It can be noticed that, for this case, the simplest classical literature models present a good fit and give a good explanation of what happens in this type of environment. On the other hand, based on the results, future directions for the research were identified. For example, it is important to obtain a mathematical model for propagation through vegetation in situations in which canalization occurs and to develop a mathematical model for propagation in agricultural environments with obstacles, considering, as a parameter, the radius of the tree trunks and their disposal on the plantation.

\section{Competing Interests}

The authors declare that there is no conflict of interests regarding the publication of this paper.

\section{Acknowledgments}

The authors would like to thank Copele, CAPES, CNPq, and Iecom for supporting this research.

\section{References}

[1] W. Maohua, "Possible adoption of precision agriculture for developing countries at the threshold of the new millennium," Computers and Electronics in Agriculture, vol. 30, no. 1-3, pp. 45-50, 2001.

[2] Aqeel-Ur-Rehman, A. Z. Abbasi, N. Islam, and Z. A. Shaikh, "A review of wireless sensors and networks' applications in agriculture," Computer Standards and Interfaces, vol. 36, no. 2, pp. 263-270, 2014.

[3] T. Wark, P. Corke, P. Sikka et al., "Transforming agriculture through pervasive wireless sensor networks," IEEE Pervasive Computing, vol. 6, no. 2, pp. 50-57, 2007.

[4] J. P. R. Lima and É. A. A. Miranda, "Fruticultura irrigada no vale do São Francisco: incorporação tecnológica, competitividade e sustentabilidade," Revista Econômica do Nordeste, vol. 32, pp. 611-632, 2001.

[5] M. S. Alencar and V. C. Rocha Jr., Communication Systems, Springer, 2005.

[6] M. D. Yacoub, Foundations of Mobile Radio Engineering, CRC Press, Boca Raton, Fla, USA, 1st edition, 1993.

[7] F. J. Pierce and T. V. Elliott, "Regional and on-farm wireless sensor networks for agricultural systems in Eastern Washington," Computers and Electronics in Agriculture, vol. 61, no. 1, pp. 3243, 2008.

[8] A. Baggio, "Wireless sensor networks in precision agriculture," Tech. Rep., Delft University of Technology, Delft, The Nederland, 2009.
[9] S. Li and H. Gao, "Propagation characteristics of $2.4 \mathrm{GHz}$ wireless channel in cornfields," in Proceedings of the IEEE 13th International Conference on Communication Technology (ICCT '11), pp. 136-140, IEEE, Jinan, China, September 2011.

[10] J. C. Giacomin, F. H. Vasconcelos, and E. J. D. Silva, "Estimating vegetation water content with wireless sensor network communication signals," in Proceedings of the IEEE Instrumentation and Measurement Technology (IMTC '07)_Synergy of Science and Technology in Instrumentation and Measurement, Warsaw, Poland, May 2007.

[11] Aqeel-ur-Rehman, Z. A. Shaikh, H. Yousuf, F. Nawaz, M. Kirmani, and S. Kiran, "Crop irrigation control using wireless sensor and actuator network (WSAN)," in Proceedings of the 2nd International Conference on Information and Emerging Technologies (ICIET '10), pp. 1-5, Karachi, Pakistan, June 2010.

[12] G. Ali, A. W. Shaikh, Aqeel-ur-Rehman, and Z. A. Shaikh, "A framework for development of cost-effective irrigation control system based on Wireless Sensor and Actuator Network (WSAN) for efficient water management," in Proceedings of the 2nd International Conference on Mechanical and Electronics Engineering (ICMEE '10), vol. 2, pp. 378-381, IEEE, Kyoto, Japan, August 2010.

[13] M. Keshtgari and A. Deljoo, "A wireless sensor network solution for precision agriculture based on zigbee technology," Wireless Sensor Network, vol. 4, no. 1, pp. 25-30, 2012.

[14] J. S. Seybold, Introduction to RF Propagation, John Wiley \& Sons, Hoboken, NJ, USA, 2005.

[15] A. Goldsmith, Wireless Communications, Cambridge University Press, New York, NY, USA, 1st edition, 2005.

[16] C. Phillips, D. Sicker, and D. Grunwald, "A survey of wireless path loss prediction and coverage mapping methods," IEEE Communications Surveys \& Tutorials, vol. 15, no. 1, pp. 255-270, 2013.

[17] Y. Dodge, The Concise Encyclopedia of Statistics, Springer, New York, NY, USA, 1st edition, 2008.

[18] W. C. Y. Lee, Mobile Communications Engineering, McGrawHill, New York, NY, USA, 1st edition, 1982.

[19] W. C. Y. Lee, Mobile Communications Design Fundamentals, John Wiley \& Sons, Hoboken, NJ, USA, 2nd edition, 1993.

[20] T. S. Rappaport, Wireless Communications: Principles and Practice, Prentice Hall, Upper Saddle River, NJ, USA, 2nd edition, 2002.

[21] M. A. Weissberger, "An initial critical summary of models for predicting the attenuation of radio waves by foliage," Tech. Rep. ECAC-TR-81-101, Eletromagnetic Compatibility Analysis Center, Annapolis, Md, USA, 1981.

[22] Y. S. Meng, Y. H. Lee, and B. C. Ng, "Empirical near ground path loss modeling in a forest at VHF and UHF bands," IEEE Transactions on Antennas and Propagation, vol. 57, no. 5, pp. 1461-1468, 2009.

[23] F. Larson, Estatística Aplicada, Pearson Prentice Hall, São Paulo, Brazil, 4th edition, 2010. 


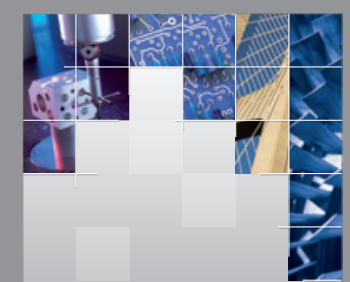

\section{Enfincering}
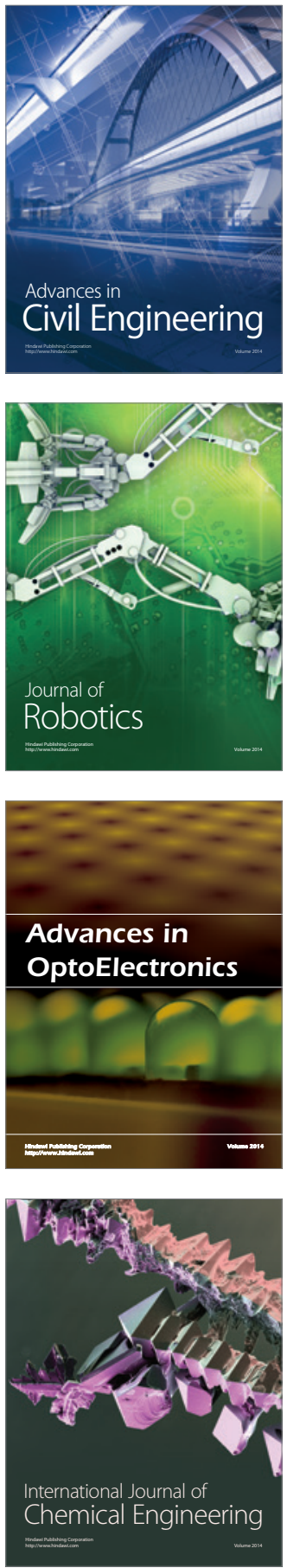

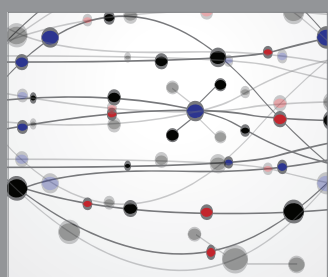

The Scientific World Journal

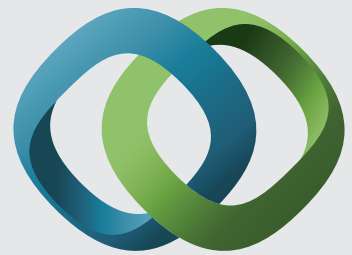

\section{Hindawi}

Submit your manuscripts at

https://www.hindawi.com
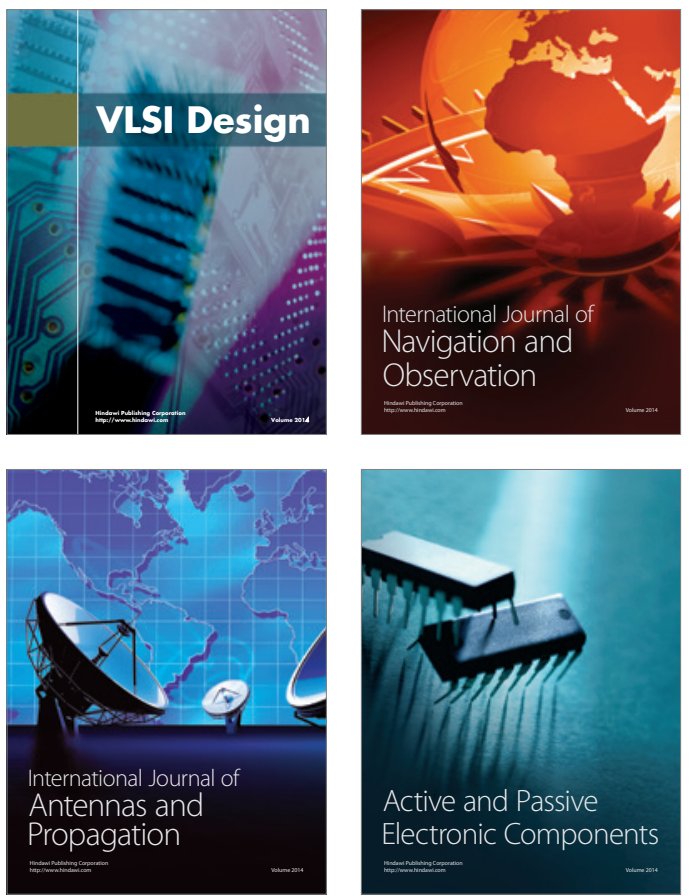
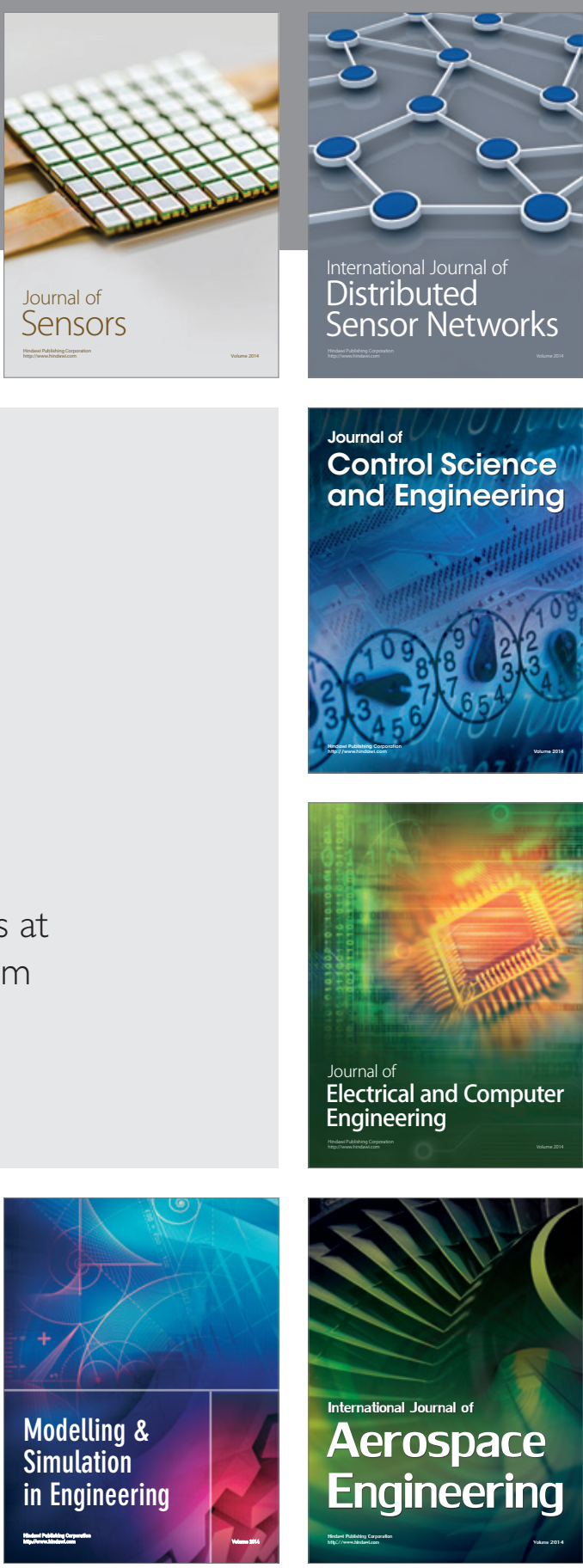

International Journal of

Distributed

Sensor Networks

$-$

Joumal of

Control Science

and Engineering
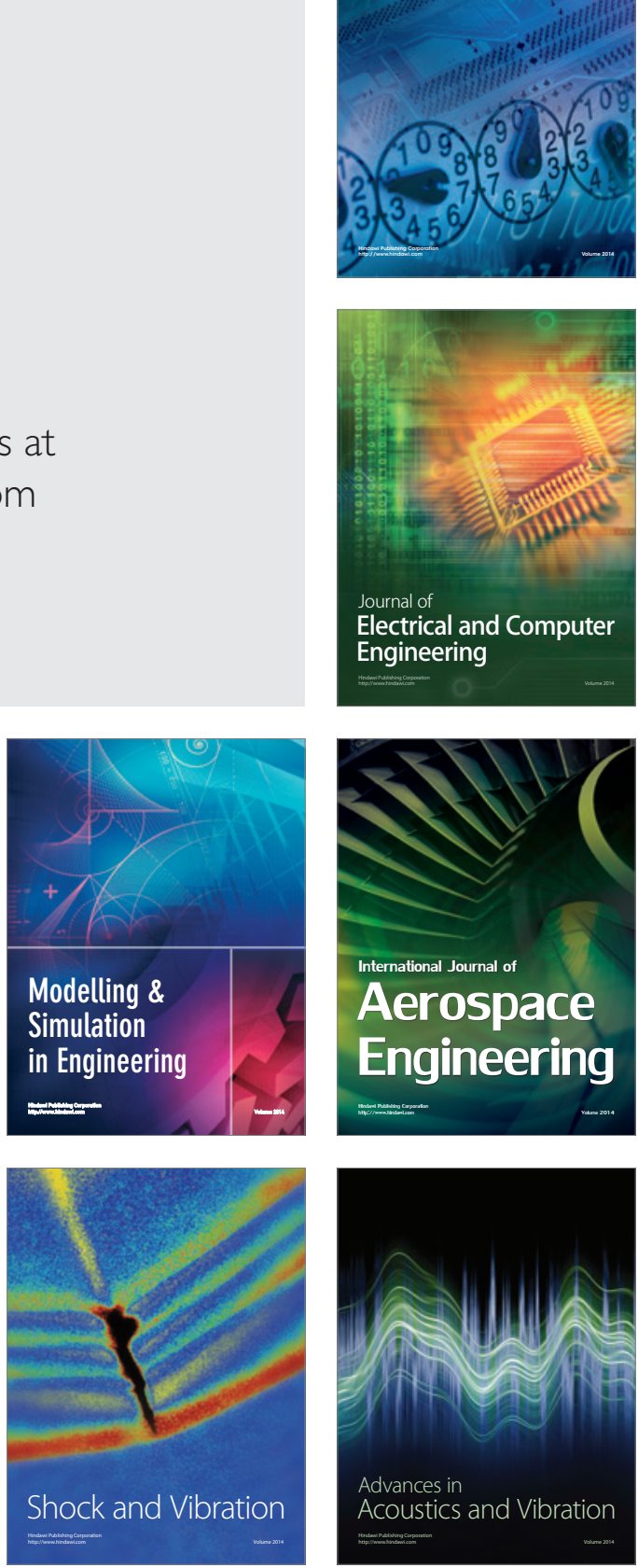